\title{
Angelica Sinensis Extract Protects Against Memory Deficits by Promoting P38 MAPK-mediated Hippocampal Neurogenesis and Neuronal Survival in the Chronic Phase of Transient Global Cerebral Ischemia in Rats
}

\section{Yu-Chen Lee}

China Medical University

Hui-Chi Huang

China Medical University

Shung-Te Kao

China Medical University

Chin-Yi Cheng ( $\nabla$ c5398@ms22.hinet.net )

School of Post-baccalaureate Chinese Medicine, College of Chinese Medicine, China Medical University, Taichung 40402, Taiwan

\section{Research}

Keywords: global cerebral ischemia, cognitive deficits, Angelica sinensis, p38 mitogen-activated protein kinase, bromodeoxyuridine, microtubule-associated protein 2, brain-derived neurotrophic factor

Posted Date: September 17th, 2020

DOl: https://doi.org/10.21203/rs.3.rs-77044/v1

License: (c) (1) This work is licensed under a Creative Commons Attribution 4.0 International License. Read Full License 


\section{Abstract}

Background: Reversible cardiac arrest and diseases related to reduced blood flow to the brain cause transient global cerebral ischemia (GCl), which may induce long-term cognitive deficits. In addition, hippocampal cornu ammonis 1 (CA1) pyramidal neurons are selectively vulnerable to ischemic insults and play an important role in memory. This study aimed to determine the effects of the Angelica sinensis (Oliv.) Diels (ASD) extract on ischemia-induced memory deficits at $28 \mathrm{~d}$ after transient global cerebral ischemia $(\mathrm{GCl})$ and to investigate the precise mechanisms underlying the p38 mitogen-activated protein kinase (MAPK)-related signaling pathway's involvement in hippocampal neurogenesis. Rats underwent 25 min of four-vessel occlusion. The ASD extract was intragastrically administered at doses of $0.25 \mathrm{~g} / \mathrm{kg}$ (ASD-0.25 g), $0.5 \mathrm{~g} / \mathrm{kg}$ (ASD-0.5 g), $1 \mathrm{~g} / \mathrm{kg}$ (ASD-1 g), $1 \mathrm{~g} / \mathrm{kg}$ after dimethyl sulfoxide administration (D+ASD-1 g), or $1 \mathrm{~g} / \mathrm{kg}$ after SB203580 (a p38 MAPK inhibitor) administration (SB+ASD-1 g) at 1, 3, 7, 10, $14,17,21$, and $24 \mathrm{~d}$ after transient $\mathrm{GCl}$.

Results: $A S D-0.5 \mathrm{~g}, A S D-1 \mathrm{~g}$, and D+ASD-1 $\mathrm{g}$ treatments effectively attenuated memory deficits and had the following effects: upregulation of bromodeoxyuridine (BrdU) and Ki67 expression, and BrdU/neuronal nuclei (NeuN) co-expression in the hippocampal dentate gyrus (DG); upregulation of microtubuleassociated protein 2 / NeuN co-expression in the hippocampal CA1 region; upregulation of phospho-p38 MAPK ( $p-p 38$ MAPK), phospho-cAMP response element-binding protein ( $p$-CREB), brain-derived neurotrophic factor (BDNF), glial cell line-derived neurotrophic factor (GDNF), and vascular endothelial growth factor A (VEGF-A) expression in the hippocampus. Furthermore, most BrdU-positive cells colocalized with NeuN and Ki67 in the hippocampal DG. SB+ASD-1 g treatment abrogated the effects of ASD-1 $\mathrm{g}$ on the expression of these proteins and exacerbated memory deficits.

Conclusion: $A S D-0.5 \mathrm{~g}$ and $A S D-1 \mathrm{~g}$ treatments provided neuroprotective effects against memory deficits by enhancing hippocampal neurogenesis and dendritic stability. The effects of the ASD extract on hippocampal neurogenesis and neuronal survival are caused by the activation of p38 MAPK-mediated CREB/BDNF, GDNF, and VEGF-A signaling pathways in the hippocampus at $28 d$ after transient GCI.

\section{Background}

Various clinical conditions, including reversible cardiac arrest and diseases related to reduced blood flow to the brain, induce transient global cerebral ischemia (GCl). Transient $\mathrm{GCl}$ frequently causes long-lasting cognitive impairments, principally memory and learning dysfunction, and contributes to the development of neurological disorders, such as vascular dementia [1, 2]. Hippocampal cornu ammonis 1 (CA1) neurons are selectively vulnerable and undergo delayed degeneration in response to ischemic injury. Furthermore, the degeneration of these pyramidal neurons results in cognitive impairment [3]. However, the pharmacological rescue of post-ischemic hippocampal neurogenesis enhances cognitive function recovery $[4,5]$. The process of adult hippocampal neurogenesis includes the proliferation, differentiation, and survival of neural progenitor cells, which are regulated by different signaling pathways following ischemic brain injury [6]. Activation of mitogen-activated protein kinase (MAPK) cascades, including 
extracellular signal-regulated kinase 1/2 and p38 MAPK, plays crucial roles in neurogenesis in the hippocampal dentate gyrus (DG) after cerebral ischemia [7]. Furthermore, various ischemic stroke models have indicated that p38 MAPK plays a dual role in the regulation of adult hippocampal neurogenesis. Studies have reported that activation of p38 MAPK suppresses neurogenesis in the ischemic cortex and hippocampus in the chronic phase of cerebral ischemia $[8,9]$, whereas other studies have reported that activation of p38 MAPK-mediated signaling promotes the differentiation of neural stem/progenitor cells (NSPCs) and neurogenesis in the ischemic area after transient [10] and permanent [11] middle cerebral artery occlusion. During neurogenesis, the MAPK signaling pathway activates the downstream transcription factor CAMP response element-binding protein (CREB), which binds to DNA, thus inducing target gene transcription, and enhancing neural progenitor cell proliferation, differentiation, and survival in the hippocampal DG, which reduces memory impairment after $\mathrm{GCl}$ [12].

The adult mammalian brain is known to continuously generate new neurons in the subventricular zone (SVZ) of the lateral ventricle and the subgranular zone (SGZ) of the hippocampal DG. Adult hippocampal progenitor cells are generated at the border between the dentate granule cell layer (GCL) and the hilus [13]. Moreover, trophic factors, such as brain-derived neurotrophic factor (BDNF), nerve growth factor (NGF), glial cell line-derived neurotrophic factor (GDNF), and vascular endothelial growth factor (VEGF), are crucial in facilitating neuronal proliferation, differentiation and survival $[14,15]$. Studies have reported that the upregulation of neurotrophic factors is responsible for the effects of MAPK signaling cascades on ischemia-induced hippocampal neurogenesis $[15,16]$. Neurogenesis principally occurs in the periinjured brain region (SVZ and SGZ), where the newborn neurons migrate, along angiogenic niches, into the existing hippocampal network and the damaged cortex. The newborn neurons subsequently provide cell replacement, thereby exerting a compensatory effect after ischemic stroke [10,17]. Thus, neurogenesis from progenitor cells occurs in the hippocampal DG as a compensatory action to brain insults, such as GCI $[1,18]$.

Bromodeoxyuridine (BrdU) is a synthetic analog of thymidine that can be incorporated into DNA in the synthesis phase of the cell cycle, and it is widely used as an indicator of cell proliferation in living tissues and as a marker of neurogenesis [18]. BrdU is expressed in the SGZ of the hippocampal DG after GCI [19]. Ki67 is a cell cycle-related protein, and it has been identified as a cell proliferation marker. Ki67 is primarily expressed in the hippocampal DG after cerebral ischemic injury [20]. Therefore, after $\mathrm{GCl}$, hippocampal progenitor cell proliferation is identified based on BrdU and Ki67 expression in the DG. The pharmacological upregulation of BrdU expression in the hippocampal DG indicates that the therapeutic intervention has triggered the self-renewal of NSPCs after ischemic stroke [21]. Microtubule-associated protein 2 (MAP-2), located in the neuronal perikarya and dendrites, is crucial in plasticity and in stabilizing the dendrite shape during neuronal development [22]. MAP-2, a neuronal dendritic marker, can be used as an early indicator for measuring the development of mature neurons in neurogenesis after cerebral ischemia [23].

Angelica sinensis (Oliv.) Diels (ASD), commonly known as Dang Gui, is a popular Chinese herb that has long been used to treat gynecological diseases, anemia, and ischemic stroke [24, 25]. The major bioactive 
compounds of ASD are ferulic acid and ligustilide [26]. Ferulic acid protects against memory deficits by downregulating the toll-like receptor 4 /myeloid differentiation factor 88 signaling pathway after transient $\mathrm{GCl}$ [27]. A study in a rat model of chronic cerebral hypoperfusion $(\mathrm{CCH})$ revealed that ligustilide exerts neuroprotective effects on memory by inhibiting apoptosis in the hippocampus [2]. The ASD extract enhances adult hippocampal neurogenesis by activating CREB/BDNF signaling in the hippocampus after $\mathrm{CCH}$ [28]. In a previous study, we determined that the ASD extract exerts neuroprotective effects against cerebral ischemia/reperfusion (I/R) injury after transient $\mathrm{GCl}$ by activating the $\mathrm{p} 38 \mathrm{MAPK}$-mediated 90kDa ribosomal 66 kinase (p90RSK)/phospho-Bad and p90RSK/CREB/BDNF signaling pathways in the hippocampus [29]. Based on the aforementioned findings, we speculated that the ASD extract protects against ischemia-induced memory impairment by regulating p38 MAPK-related signaling in the hippocampus in the chronic phase of transient GCl.

Therefore, we aimed to determine the effects of the ASD extract on hippocampal neurogenesis and neuronal survival at $28 \mathrm{~d}$ after transient $\mathrm{GCl}$ and to investigate the precise mechanisms underlying p38 MAPK-related signaling in the hippocampus.

\section{Methods}

\section{Experimental animals}

Adult male Sprague-Dawley rats (300-350 g) were purchased from Lasco Co. (Taiwan). All rats were housed under standard laboratory conditions and were maintained in a constant $12: 12 \mathrm{~h}$ light-dark cycle, at a controlled temperature of $22-24{ }^{\circ} \mathrm{C}$, and in relative humidity of $55 \%$. All study procedures were performed following the guidelines approved by the Institutional Animal Care and Use Committee of China Medical University (Permit Number: 2018-075). All surgical procedures were performed under appropriate anesthesia to avoid or minimize discomfort, pain, and stress in the animals.

\section{ASD extract preparation}

ASD extracts, with ASD powders (Batch Number A1306801), were purchased from Chuang Song Zong Pharmaceutical Co., Ltd. (Kaohsiung, Taiwan). ASD extract powder was prepared as previously described [29]. In brief, the dried roots of ASD were extracted using boiling water for $1.5 \mathrm{~h}$ and then the aqueous extract and essential oils from ASD were collected. The concentrated aqueous extract, essential oils, and excipients including microcrystalline cellulose and corn starch were placed in the fluidized bed dryer and granulated using fluidized bed granulation techniques. Each gram of the ASD powder consists of $0.5 \mathrm{~g}$ of the DG extract, $0.4 \mathrm{~g}$ of microcrystalline cellulose, and $0.1 \mathrm{~g}$ of corn starch. In this study, $2 \mathrm{~g}$ of the ASD extract powder was dissolved in $8 \mathrm{~mL}$ of normal saline. The supernatant fraction of the ASD extract was subsequently obtained by centrifugation at $1000 \times \mathrm{g}$ at $4{ }^{\circ} \mathrm{C}$ for $10 \mathrm{~min}$. The final concentration of the ASD aqueous extract was $0.125 \mathrm{~g} / \mathrm{mL}$.

\section{Assessment of ASD extract indicators using ultra-performance liquid chromatography}


The indicator compounds of the ASD extract, including ferulic acid and ligustilide, were detected using an Agilent ultra-performance liquid chromatography (UPLC) system (Agilent 1260 Infinity II, Agilent Technologies, Inc.). Ferulic acid (purity $>99 \%$, Sigma-Aldrich, St. Louis, MO, USA) and ligustilide (purity > $98 \%$, Adooq BioScience, Irvine, CA, USA) were accurately weighted and subsequently dissolved in methanol to prepare the standard solutions. A series of standard solutions were prepared with concentrations of $1,0.5,0.25,0.1,0.05,0.025$, and $0.01 \mathrm{mg} / \mathrm{mL}$. The aqueous extract of ASD $(0.125$ $\mathrm{g} / \mathrm{mL}$ ) was prepared as the test solution. One microliter of the standard solution or $3 \mu \mathrm{L}$ of the test solution was injected using an autosampler (Agilent 1260 Infinity II Vialsampler). The UPLC profile of the ASD extract was performed using a RP-18 column (Poroshell 120 EC-C18, $3.0 \times 100$ mm. I.D., $2.7 \mu \mathrm{m}$ ). The mobile phase consisted of a mixture of acetonitrile $(A)$ and $0.03 \%$ phosphoric acid (B) solution. Gradient elution was performed as follows: 0-12 min (phase A: 5\%-70\%), 12-13 min (phase A: 70\%$80 \%$ ), and $13-15 \mathrm{~min}$ (phase $\mathrm{A}: 80 \%-100 \%$ ). The flow rate was $0.5 \mathrm{~mL} / \mathrm{min}$ and the ultraviolet detection wavelength was set at $320 \mathrm{~nm}$.

\section{Transient GCI}

$\mathrm{GCl}$ was induced in the rats using the 4-vessel occlusion (4-VO) method as previously described [30]. In brief, the rats were anesthetized by inhalation of $5 \%$ isoflurane, and the anesthesia was maintained using $2 \%$ isoflurane. A dorsal neck midline incision was performed to expose the alar foramina of the first cervical vertebrae, and the bilateral vertebral arteries were permanently occluded using electrocautery. Twenty-four hours later, another incision was performed on the ventral neck to expose the bilateral common carotid arteries (CCAs), which were occluded for 25 min using vascular clips. The rats that displayed the loss of righting reflex and whose pupils were dilated during the ischemic period were validated as successful 4 -VO models and were included in this study.

\section{Passive avoidance task}

A passive avoidance task was performed to evaluate 4-V0-induced memory deficits; the task was a modification of previously described procedures [31]. The passive avoidance apparatus (San Diego Instruments, San Diego, CA, USA) consisted of a box with two equal-sized light/dark compartments (25 $\times$ $20 \times 17 \mathrm{~cm}^{3}$ each), which were separated by a guillotine-type door $\left(9 \times 7 \mathrm{~cm}^{2}\right)$. The floor of each compartment consisted of 14 stainless steel rods. The floor rods of the dark compartment administered a brief electric shock. The memory evaluation test consisted of training and retention protocols. The training protocol comprised two acquisition tests $1 \mathrm{~h}$ before surgery. In the first acquisition test, the rats were individually placed in the light compartment for a 30-s habituation period. The guillotine door was then raised; when the rat entered the dark compartment, the door was immediately closed and a brief electric foot shock $(50 \mathrm{~Hz}, 0.5 \mathrm{~mA}, 3 \mathrm{~s})$ was delivered. The training was completed when the rats no longer entered the dark compartment before the cut-off time (120 s). The retention protocol was performed without foot shock at 7, 14, 21, and $28 \mathrm{~d}$ after reperfusion. The latency to enter the dark compartment (step-through latency [STL]) was measured, and the maximum score was recorded as 300 s. 


\section{Experiment A}

\section{Grouping}

The rats were randomly divided into seven groups ( $\mathrm{n}=4-5)$ : Sham, Control, ASD- $0.25 \mathrm{~g}$, ASD-0.5 g, ASD-1 $\mathrm{g}, \mathrm{D}+\mathrm{ASD}-1 \mathrm{~g}$, and SB+ASD-1 g groups. The rats in the ASD- $0.25 \mathrm{~g}$, ASD-0.5 g, and ASD-1 g groups were intragastrically administered $0.25 \mathrm{~g} / \mathrm{kg}, 0.5 \mathrm{~g} / \mathrm{kg}$, and $1 \mathrm{~g} / \mathrm{kg}$ of the ASD extract, respectively, at $1,3,7,10$, $14,17,21$, and $24 \mathrm{~d}$ after transient $\mathrm{GCl}$. Furthermore, to determine the rate of progenitor proliferation, the rats received daily intraperitoneal injections of $\mathrm{BrdU}$ ( $50 \mathrm{mg} / \mathrm{kg}$, Sigma-Aldrich) from day 14 to day 27 after reperfusion. On day 28 after reperfusion, the rats underwent $\mathrm{CO}_{2}$ euthanasia until 1 min after

breathing had stopped and their brains were quickly removed. The rats in the D+ASD-1 g group underwent the same experimental procedures as those in the ASD-1 g group, but they were intracerebroventricularly (ICV) administered 1\% dimethyl sulfoxide (DMSO) before the bilateral CCA occlusion. The rats in the SB+ASD-1 g group underwent the same experimental procedures as those in the D+ASD-1 g group, but they were ICV administered SB203580, a selective inhibitor of p38 MAPK, instead of 1\% DMSO. The rats in the Control group underwent the same experimental procedures as those in the ASD-1 g group, but the ASD extract was substituted by saline solution. The rats in the Sham group underwent the same experimental procedures as those in the Control groups, but their bilateral CCAs were only exposed instead of occluded.

\section{ICV administration of SB203580 or $1 \%$ DMSO}

The rats were ICV administered SB203580 or $1 \%$ DMSO after anesthesia maintenance through isoflurane inhalation, and two symmetrical burr holes were drilled on both sides of the parietal bone. The burr holes were $2 \mathrm{~mm}$ lateral, $3 \mathrm{~mm}$ posterior to the bregma, and $3.5 \mathrm{~mm}$ under the cortical surface. The rats were ICV administered $10 \mu \mathrm{L}$ SB203580 (2 mM, \#S1076 Selleckchem.com) or 1\% DMSO solution through each burr hole. ICV administration of the solution was performed using a microliter syringe (10 $\mu \mathrm{L}$, Hamilton Company, Reno, NV, USA).

\section{Assessment of immunofluorescence staining}

The rats were euthanized by inhalation of $\mathrm{CO}_{2}$ at $28 \mathrm{~d}$ after reperfusion, and their brains were rapidly removed. The brain tissue sections were prepared as previously described [29]. Coronal brain sections were fixed with $4 \%$ paraformaldehyde for $15 \mathrm{~min}$ at room temperature (RT). After the brain sections were washed thrice with phosphate-buffered saline/0.01\% Tween 20 (PBST), the sections were incubated with $1 \%$ Triton X-100 for 30 min at RT. The sections were incubated with $1 \%$ bovine serum albumin for $1 \mathrm{~h}$ at RT and were subsequently exposed to rabbit (1:100 dilution) and mouse (1:100 dilution) primary antibodies (listed in Table 1) overnight at $4{ }^{\circ} \mathrm{C}$. After the sections were washed thrice with PBST, they were incubated with anti-rabbit (1:200 dilution) and anti-mouse (1:200 dilution) secondary antibodies for $1.5 \mathrm{~h}$ at $37^{\circ} \mathrm{C}$. The sections were then counterstained with 4',6-diamidino-2-phenylindole (DAPI, ab4139 abcam) and mounted using an aqueous mounting medium. The immunopositive cells in the selected 
hippocampal SGZ or CA1 regions were detected in each of three $400 \times$ magnification fields under a fluorescence microscope (CKX53, Olympus, Tokyo, Japan).

\section{Assessment of immunohistochemistry}

The brain tissue sections were prepared, and subsequent immunohistochemistry (IHC) evaluations were performed as previously described [32]. Anti-ki67 antibody used in the IHC evaluation was listed in Table 1. Immunopositive cells in the hippocampal SGZ region were calculated in each of three $400 x$ magnification fields using a light microscope (Axioskop 40, Zeiss, Oerzen, Germany). For the negative control, the brain section in the ASD-1 g group was incubated without Ki67 primary antibody.

\section{Experiment B}

\section{Grouping}

The rats were randomly divided into seven groups $(n=4)$ : Sham, Control, ASD- $0.25 \mathrm{~g}$, ASD-0.5 g, ASD-1 g, D+ASD-1 g, and SB+ASD-1 g groups. These groups underwent the same procedures as those performed in Experiment $A$.

\section{Evaluation of Western blot}

The rats underwent $\mathrm{CO}_{2}$ euthanasia at $28 \mathrm{~d}$ after reperfusion, and their brains were immediately removed. The right and left hippocampal tissues were carefully separated, collected, and homogenized on ice. The protein concentrations of the hippocampal tissues were measured using a Bio-Rad protein assay. The proteins separated by gel electrophoresis, transfer onto nitrocellulose membranes, and densitometric analysis were performed as previously described [33]. The primary antibodies used in the Western blot analyses were listed in Table 1.

\section{Statistical analysis}

All the results are expressed as means \pm standard deviations. Statistical analysis of the data obtained for the experiment groups from SLT, immunofluorescence (IF), IHC, and Western blot analyses was performed using one-way analysis of variance followed by Scheffe's post-hoc test. Significant differences were accepted for $P<0.05$.

\section{Results}

\section{UPLC analysis of the ASD extract}

After the detection of the standard and ASD extract solutions through UPLC, the retention times $\left(T_{R}\right)$ of ferulic acid and ligustilide were revealed at $5.87 \mathrm{~min}$ and $12.81 \mathrm{~min}$, respectively. The concentrations of ferulic acid and ligustilide in the ASD extract solution were $0.234 \mathrm{mg} / \mathrm{mL}$ and $0.027 \mathrm{mg} / \mathrm{mL}$, respectively (Figs. 1A-1C). 


\section{Effects of ASD extract treatments on memory deficits}

The STL was lower in the Control group than in the Sham group (all $P<0.05$ ) and was greater in the ASD$0.5 \mathrm{~g}$, ASD-1 g, and D+ASD-1 g groups than that in the Control group (all $P<0.05$; Fig. 2) at 7, 14, 21, and $28 \mathrm{~d}$ after reperfusion. However, the STL did not differ significantly between the Control, ASD-0.25 g, and SB+ASD-1 g groups $(P>0.05)$.

\section{Effects of ASD extract treatments on BrdU expression and BrdU/neuronal nuclei co-expression}

Fewer BrdU-positive and BrdU/neuronal nuclei (NeuN)-positive cells were identified in the SGZ of the hippocampal DGs (Fig. 3B) of the Control group compared with the Sham group (both $P<0.05$ ) and more positive cells were identified in the ASD-0.5 g, ASD-1 g, and D+ASD-1 g groups compared with the Control group at $28 \mathrm{~d}$ after reperfusion (all $P<0.05$; Figs. 3A, 3C, and 3D). The numbers of BrdU-positive and BrdU/NeuN-positive cells in the hippocampal SGZs did not differ significantly between the Control, ASD$0.25 \mathrm{~g}$, and SB+ASD-1 g groups (all $P>0.05$ ). The majority of BrdU-positive cells colocalized with NeuN in the hippocampal SGZ.

\section{Effects of ASD extract treatments on Ki67 expression}

Fewer Ki67-positive cells were identified in the hippocampal SGZ (Fig. 4C) of the Control group compared with the Sham group $(P<0.05)$, and higher numbers were observed in the ASD-0.5 g, ASD-1 g, and D+ASD-1 g groups compared with the Control group at $28 \mathrm{~d}$ after reperfusion (all $P<0.05$; Figs. $4 \mathrm{~A}$ and 4D). However, the number of Ki67-positive cells in the hippocampal SGZ did not differ significantly between the Control, ASD-0.25 g, and SB+ASD-1 g groups (all $P>0.05$ ). Furthermore, BrdU-positive cells colocalized with Ki67 in the hippocampal SGZ (Figs. 4E-1-E-3).

\section{Effects of ASD extract treatments on MAP-2/NeuN co-expression}

Fewer MAP-2/NeuN-positive cells were identified in the CA1 region (Fig. 5B) of the Control group compared with the Sham group $(P<0.05)$, and more MAP-2/NeuN-positive cells were identified in the ASD-0.5 g, ASD-1 g, and D+ASD-1 g groups compared with the Control group at $28 \mathrm{~d}$ after reperfusion (all $P<0.05$; Figs. 5A and 5C). The number of MAP-2/NeuN-positive cells in the CA1 region did not differ significantly between the Control, ASD-0.25 g, and SB+ASD-1 g groups (all $P>0.05$ ).

\section{Effects of ASD extract treatments on p-p38 MAPK, p38 MAPK, p-CREB, CREB, BDNF, NGF, GDNF, and VEGF-A expression}

The ratios of p-p38 MAPK/p38 MAPK, p-CREB/CREB, BDNF/actin, GDNF/actin, and VEGF-A/actin in the hippocampus were lower in the Control group (0.4-fold, 0.4-fold, 0.3-fold, 0.4-fold, and 0.4-fold, respectively) compared with the Sham group (all $P<0.05$ ) and were greater in the ASD-0.5 g (2.5-fold, 2.3fold, 4.2-fold, 2.4-fold, and 2.7-fold, respectively), ASD-1 g (2.9-fold, 2.1-fold, 4.1-fold, 2.4-fold, and 2.6fold, respectively), and D+ASD-1 g (2.6-fold, 2.3-fold, 4.2-fold, 2.6-fold, and 2.8-fold, respectively) groups compared with the Control group at $28 \mathrm{~d}$ after reperfusion (all $P<0.05$; Figs. 6A-6D, 7A, 7C, and 7D). 
However, the ratios of these proteins did not differ significantly between the Control, ASD-0.25 $\mathrm{g}$, and SB+ASD-1 g groups $(P>0.05)$. Furthermore, no significant differences were identified in the hippocampal NGF/actin ratios in the experimental groups ( $P>0.05$; Figs. 7A and 7B).

\section{Discussion}

In a previous study, we determined that ASD extract treatments protect against I/R injury by activating p38 MAPK-mediated signaling in the hippocampus at $7 \mathrm{~d}$ after transient $\mathrm{GCl}$ [29]. In the present study, we extended our research to the chronic phase $(28 \mathrm{~d})$ of $\mathrm{GCl}$ to clarify the relationship between p38 MAPK-related signaling and adult hippocampal neurogenesis. Various ischemic models have indicated that the p38 MAPK-mediated signaling pathway plays diverse roles in ischemia-induced cognitive impairment. Studies have reported that pharmacological treatments promote functional recovery of memory deficits by suppressing p38 MAPK-mediated inflammatory cascades in the acute [34] and chronic [9] phases of transient cerebral ischemia. However, our previous findings revealed that activation of p38 MAPK/p90RSK-related signaling pathways in the hippocampus provides neuroprotective effects against I/R injury in the subacute phase of transient $\mathrm{GCl}$ [29]. In the present study, the rats underwent 25 min of 4-VO, resulting in significant memory deficits at 7-28 $\mathrm{d}$ after transient cerebral ischemia. The ASD extract administered at doses of $0.5 \mathrm{~g} / \mathrm{kg}$ (ASD-0.5 g), $1 \mathrm{~g} / \mathrm{kg}$ (ASD- $1 \mathrm{~g}$ ), and $1 \mathrm{~g} / \mathrm{kg}$ after ICV injections of $1 \%$ DMSO (D+ASD-1 g) effectively attenuated memory impairment; administering $0.25 \mathrm{~g} / \mathrm{kg}$ (ASD-0.25 g) did not attenuate memory impairments. However, ICV injections of SB203580, a selective inhibitor of p38 MAPK, (SB+ASD-1 g) completely abrogated the effects of the ASD extract (ASD-1 g) on promoting the functional recovery of memory. Therefore, activation of p38 MAPK in the hippocampus may have a key function in restoring memory loss in the chronic phase of transient $\mathrm{GCl}$. Increasing evidence indicates that hippocampal neurogenesis originating from progenitor cells in the DG region is closely related to the restoration of the ischemia-induced memory deficits following transient $\mathrm{GCI}[3,35]$. Furthermore, activation of the p38 MAPK-related pathways enhances the survival and differentiation of newborn neurons, inducing increased neurogenesis in the ischemic areas after ischemic stroke $[10,11]$. Therefore, the effects of ASD extract treatments (ASD-0.5 g and ASD-1 g) on memory dysfunction may be, at least partially, caused by the promotion of p38 MAPK-mediated adult hippocampal neurogenesis in the chronic phase of transient GCl.

Adult hippocampal neurogenesis is a complex multistep process that includes the proliferation and differentiation of neurons to generate new excitatory granule cells; the process originates from progenitor cells in the SGZ of the DG and culminates in various signaling events triggered by stimuli [36]. During neurogenesis, progenitor cells proliferate in the SGZ of the hippocampus and subsequently differentiate into neurons or glial cells [37]. The thymidine analog BrdU is incorporated in the newly synthesized DNA of replicating cells and is commonly used to detect neural progenitor cell proliferation in the central nervous system (CNS) [38]. Studies have demonstrated that most BrdU-labeled cells colocalize with the neuronal marker NeuN in the hippocampal SGZ in the chronic phase of transient GCl. Furthermore, $\mathrm{BrdU} / \mathrm{NeuN}$ double-labeled cells represent newborn neurons, which are closely associated with recovery of hippocampal function [19, 39]. Ki67, a mitosis-related protein expressed in dividing cells, exhibits a 
similar expression pattern to BrdU. Therefore, $\mathrm{Ki} 67$ is also widely used as a cell proliferation marker. Moreover, Ki67 is primarily applied as a diagnostic tool in the study of CNS tumors [20,40]. Studies have reported that the pharmacological promotion of BrdU-associated and Ki67-associated neural progenitor cell proliferation in the SGZ of the DG exerts neuroprotective effects against memory dysfunction in the chronic phase of GCI [41, 42]. The IF, IHC, and Western blot results of the present study indicated that the majority of BrdU-positive cells were double-labeled with NeuN and Ki67, and that the numbers of BrdU-, $\mathrm{BrdU} / \mathrm{NeuN}$ - and Ki67-positive cells were downregulated in the SGZ of the DG. ASD extract treatments effectively restored the reduced numbers of these immunopositive cells in the SGZ of the DG at $28 \mathrm{~d}$ after GCl. However, SB+ASD-1 g treatment significantly reduced the effects of ASD-1 g on p38 MAPK activation, and increased BrdU, BrdU/NeuN, and Ki67 expression in the hippocampus in the chronic phase of $\mathrm{GCl}$. These results demonstrated that ASD extract treatments effectively promote newborn neuron and BrdU/Ki67-mediated progenitor cell proliferation in the SGZ of the DG at $28 \mathrm{~d}$ after transient $\mathrm{GCl}$ by activating the p38 MAPK-related signaling pathway in the hippocampus.

MAP-2, a marker of neuronal dendrites, stabilizes the microtubule cytoskeleton of dendrites and is a sensitive and early indicator for evaluating dendritic integrity, neuronal damage, and neuron maturity in post-ischemia neurogenesis [2, 23, 43]. In the process of neurogenesis, BrdU/MAP-2 double-labeled cells are generated in the $S G Z$ and migrate to the $\mathrm{GCL}$, where they become mature neurons, whereas reduced MAP-2 expression in the hippocampal CA1 region indicates neuronal damage or death $[43,44]$. Increased MAP-2 expression in the CA1 region promotes hippocampal dendrite and synapse stability, which alleviates memory dysfunction in the chronic phase of $\mathrm{GCI}[43,45]$. In the hippocampal CA1 region, MAP2-positive cells co-labeling with NeuN indicate dendritic outgrowth of hippocampal pyramidal neurons. In addition, NeuN, a marker of mature neuron, can be used to determine the survival of neuron following ischemic insults [46]. In the present study, IF double staining revealed that the expression of MAP-2/NeuN was downregulated in the hippocampal CA1 regions. However, $28 \mathrm{~d}$ after transient GCl, ASD extract treatments effectively restored MAP-2/NeuN expression. These findings further indicate that ASD treatments alleviate neuronal damage and enhance the dendritic outgrowth recovery of injured neurons in the CA1 region, thereby promoting neuronal survival. However, SB+ASD-1 g treatment markedly reduced the effects of ASD-1 g on hippocampal neuronal survival. These results indicate that ASD extract treatments provide neuroprotective effects against memory deficits by promoting dendritic stability in hippocampal pyramidal neurons. Furthermore, the effects of ASD extract treatments on hippocampal neuronal survival and dendritic stability at $28 \mathrm{~d}$ after transient $\mathrm{GCl}$ are caused by the activation of the p38 MAPK-mediated signaling pathway in the hippocampus.

Studies have demonstrated that the p38 MAPK signaling pathway activates the transcription factor CREB, which regulates several downstream genes and provides anti-apoptotic neuroprotective effects in the penumbra following transient focal cerebral ischemia $[47,48]$. Our previous study indicated that pharmacological activation of p38 MAPK/CREB signaling in the hippocampus protects against I/R injury in the subacute phase of transient GCI [29]. CREB activation plays a crucial role in adult hippocampal neurogenesis, including cell proliferation, differentiation, maturation, and survival, and hippocampal dendritic-synaptic plasticity, which facilitate memory recovery after cerebral ischemia $[49,50]$. After $\mathrm{GCl}$, 
BDNF and NGF, the downstream targets of CREB, enhance the proliferation and differentiation of progenitor cells, accelerate the maturation of newborn neurons, increase neuronal survival, and promote hippocampal synaptic formation and plasticity, resulting in an increase in adult hippocampal neurogenesis and the recovery of hippocampus-dependent memory capacity $[49,51,52]$. Increased GDNF expression promotes the proliferation of glial and neuronal precursors in the hippocampus, and the effects of GDNF on progenitor cell proliferation may be crucial for stimulating neurogenesis in the hippocampal DG and for facilitating memory recovery [53]. VEGF family members include VEGF-A, VEGF$B$, VEGF-C, VEGF-D, and placental growth factor. VEGF-A exerts beneficial effects on angiogenesis and neurogenesis during cerebral ischemia [54]. Increases in VEGF-A levels promote newborn neuron proliferation and survival in the SGZ of the hippocampal DG and facilitates the migration of nascent neurons to the penumbral cortex, resulting in improvements to memory and brain injury recovery in the chronic phase of ischemic stroke [54,55]. Our experimental data revealed that the expression of p-p38 MAPK, p-CREB, BDNF, GDNF, and VEGF-A was considerably downregulated in the hippocampus, whereas ASD extract treatments considerably upregulated the expression of these proteins in the hippocampus at $28 \mathrm{~d}$ after transient GCl. Moreover, SB+ASD-1 g treatment abrogated the effects of ASD-1 g upregulation on p-p38 MAPK, p-CREB, BDNF, GDNF, and VEGF-A expression in the hippocampus. These results suggest that ASD extract treatments promote hippocampal neurogenesis and neuronal survival, thereby reducing memory impairments, by activating p38 MAPK-mediated CREB/BDNF, GDNF, and VEGF-A, but not $\mathrm{CREB} / \mathrm{NGF}$, signaling pathways in the hippocampus at $28 \mathrm{~d}$ after transient $\mathrm{GCl}$.

\section{Conclusions}

ASD extracts administered at doses of 0.5 and $1 \mathrm{~g} / \mathrm{kg}$ effectively attenuated memory deficits during $\mathrm{GCl}$. ASD extract treatments exhibited neuroprotective effects against ischemia-induced memory impairment by promoting hippocampal neurogenesis and dendritic stability. Moreover, the effects of ASD extract treatments on adult hippocampal neurogenesis and neuronal survival were revealed to be caused by activation of the p38 MAPK-mediated CREB/BDNF, GDNF, and VEGF-A signaling pathways in the hippocampus at $28 \mathrm{~d}$ after transient $\mathrm{GCl}$. Therefore, $A$. sinensis could be a therapeutic candidate for the treatment of memory deficits in the chronic phase of ischemic stroke. However, additional studies are needed to clarify the interactions of the BDNF, GDNF, and VEGF-A signaling pathways during transient $\mathrm{GCl}$.

\section{Abbreviations}

$\mathrm{GCl}$

global cerebral ischemia; CA1:cornu ammonis 1; MAPK:mitogen-activated protein kinase; DG:dentate gyrus; NSPC:neural stem/progenitor cell; CREB:CAMP response element-binding protein; SVZ:subventricular zone; SGZ:subgranular zone; GCL:granule cell layer; BDNF:brain-derived neurotrophic factor; NGF:nerve growth factor; GDNF:glial cell line-derived neurotrophic factor; VEGF:vascular endothelial growth factor; BrdU:bromodeoxyuridine; MAP-2:microtubule-associated protein 2; 
ASD:Angelica sinensis (Oliv.) Diels; CCH:chronic cerebral hypoperfusion; I/R:ischemia/reperfusion; p90RSK:90-kDa ribosomal S6 kinase; UPLC:ultra-performance liquid chromatography; 4-V0:4-vessel occlusion; CCA:common carotid artery; STL:step-through latency; ICV:intracerebroventricularly; DMSO:dimethyl sulfoxide; RT:room temperature; PBST:phosphate-buffered saline/0.01\% Tween 20; IHC:immunohistochemistry; IF:immunofluorescence; $T_{R}$ :retention time; NeuN:neuronal nuclei; CNS:central nervous system; mAU:mini absorbance unit.

\section{Declarations}

\section{Ethics approval and consent to participate}

All study procedures were performed following the guidelines approved by the Institutional Animal Care and Use Committee of China Medical University (Permit Number: 2018-075).

\section{Consent for publication}

Not applicable

\section{Availability of data and materials}

The datasets used and/or analysed during the current study are available from the corresponding author upon request.

\section{Conflicts of interest}

The authors declare that they have no competing interests.

\section{Funding}

This research was funded by the Ministry of Science and Technology of Taiwan (MOST 107-2320-B-039027-) and China Medical University Hospital (DMR-109-010), Taichung, Taiwan. These funding sources had no role in the design of the study and collection, analysis, and interpretation of the data and in writing the manuscript.

\section{Authors' Contributions}

YCL and CYC designed the study protocols. CYC performed the experiments, collected and analyzed the experimental data, and wrote the manuscript. $\mathrm{HCH}$ and STK participated in the discussions of the research works and helped to draft the manuscript. All authors read and approved the final manuscript.

\section{Acknowledgments}

We thank Miss Chun-Ting Lin and Miss Yi-Hsien Yeh at China Medical University, Taichung, Taiwan, for kindly helping with UPLC analysis. 


\section{Author details}

${ }^{1}$ Department of Chinese Medicine, China Medical University Hospital, Taichung 42056, Taiwan

${ }^{2}$ Research Center for Chinese Medicine \& Acupuncture, China Medical University, Taichung 40402, Taiwan

${ }^{3}$ Graduate Institute of Acupuncture Science, China Medical University, Taichung 40402, Taiwan

${ }^{4}$ Department of Chinese Pharmaceutical Sciences and Chinese Medicine Resources, College of Chinese Medicine, China Medical University, Taichung 40402, Taiwan

${ }^{5}$ School of Chinese Medicine, College of Chinese Medicine, China Medical University, Taichung 40402, Taiwan

${ }^{6}$ School of Post-baccalaureate Chinese Medicine, College of Chinese Medicine, China Medical University, Taichung 40402, Taiwan

${ }^{7}$ Department of Chinese Medicine, Hui-Sheng Hospital, Taichung 42056, Taiwan

\section{References}

1. de Oliveira JN, Reis LO, Ferreira EDF, Godinho J, Bacarin CC, Soares LM, et al. Postischemic fish oil treatment confers task-dependent memory recovery. Physiol Behav. 2017;177:196-207.

2. Feng Z, Lu Y, Wu X, Zhao P, Li J, Peng B, et al. Ligustilide alleviates brain damage and improves cognitive function in rats of chronic cerebral hypoperfusion. J Ethnopharmacol. 2012;144:313-21.

3. Bendel O, Bueters T, von Euler M, Ove Ogren S, Sandin J, von Euler G. Reappearance of hippocampal CA1 neurons after ischemia is associated with recovery of learning and memory. J Cereb Blood Flow Metab. 2005;25:1586-95.

4. Khodanovich M, Kisel A, Kudabaeva M, Chernysheva G, Smolyakova V, Krutenkova E, et al. Effects of Fluoxetine on Hippocampal Neurogenesis and Neuroprotection in the Model of Global Cerebral Ischemia in Rats. Int J Mol Sci. 2018;19:162.

5. Yan XB, Hou HL, Wu LM, Liu J, Zhou JN. Lithium regulates hippocampal neurogenesis by ERK pathway and facilitates recovery of spatial learning and memory in rats after transient global cerebral ischemia. Neuropharmacology. 2007;53:487-95.

6. Kwon KJ, Kim MK, Lee EJ, Kim JN, Choi BR, Kim SY, et al. Effects of donepezil, an acetylcholinesterase inhibitor, on neurogenesis in a rat model of vascular dementia. J Neurol Sci. 2014;347:66-77.

7. Tsai YW, Yang YR, Wang PS, Wang RY. Intermittent hypoxia after transient focal ischemia induces hippocampal neurogenesis and c-Fos expression and reverses spatial memory deficits in rats. PloS one. 2011;6:e24001. 
8. Bai YY, Wang L, Chang D, Zhao Z, Lu CQ, Wang G, et al. Synergistic Effects of Transplanted Endothelial Progenitor Cells and RWJ 67657 in Diabetic Ischemic Stroke Models. Stroke. 2015;46:1938-46.

9. Xuan AG, Chen Y, Long DH, Zhang M, Ji WD, Zhang WJ, et al. PPARalpha Agonist Fenofibrate Ameliorates Learning and Memory Deficits in Rats Following Global Cerebral Ischemia. Mol Neurobiol. 2015;52:601-09.

10. Song J, Cho KJ, Cheon SY, Kim SH, Park KA, Lee WT, et al. Apoptosis signal-regulating kinase 1 (ASK1) is linked to neural stem cell differentiation after ischemic brain injury. Exp Mol Med. 2013;45:e69.

11. Li Y, Huang J, He X, Tang G, Tang YH, Liu Y, et al. Postacute stromal cell-derived factor-1alpha expression promotes neurovascular recovery in ischemic mice. Stroke. 2014;45:1822-29.

12. Lee HT, Chang YC, Tu YF, Huang CC. CREB activation mediates VEGF-A's protection of neurons and cerebral vascular endothelial cells. J Neurochem. 2010;113:79-91.

13. Pforte $C$, Henrich-Noack P, Baldauf K, Reymann KG. Increase in proliferation and gliogenesis but decrease of early neurogenesis in the rat forebrain shortly after transient global ischemia. Neuroscience. 2005;136:1133-46.

14. Yoshikawa G, Momiyama T, Oya S, Takai K, Tanaka J, Higashiyama S, et al. Induction of striatal neurogenesis and generation of region-specific functional mature neurons after ischemia by growth factors. Laboratory investigation. J Neurosurg. 2010;113:835-50.

15. Karelina K, Liu Y, Alzate-Correa D, Wheaton KL, Hoyt KR, Arthur JS, et al. Mitogen and stress-activated kinases $1 / 2$ regulate ischemia-induced hippocampal progenitor cell proliferation and neurogenesis. Neuroscience. 2015;285:292-302.

16. Kim SJ, Son TG, Park HR, Park M, Kim MS, Kim HS, et al. Curcumin stimulates proliferation of embryonic neural progenitor cells and neurogenesis in the adult hippocampus. J Biol Chem. 2008;283:14497-505.

17. Rodriguez-Grande B, Varghese L, Molina-Holgado F, Rajkovic O, Garlanda C, Denes A, et al. Pentraxin 3 mediates neurogenesis and angiogenesis after cerebral ischaemia. J Neuroinflammation. $2015 ; 12: 15$.

18. Tonchev AB, Yamashima T, Zhao L, Okano H. Differential proliferative response in the postischemic hippocampus, temporal cortex, and olfactory bulb of young adult macaque monkeys. Glia. 2003;42:209-24.

19. Yagita Y, Kitagawa K, Ohtsuki T, Takasawa K, Miyata T, Okano H, et al. Neurogenesis by progenitor cells in the ischemic adult rat hippocampus. Stroke. 2001;32:1890-96.

20. Lu G, Wai SM, Poon WS, Yew DT. Ki67 and doublecortin positive cells in the human prefrontal cortices of normal aging and vascular dementia. Microsc Res Tech. 2005;68:255-7.

21. Wu H, Fan J, Zhu L, Liu S, Wu Y, Zhao T, et al. Sema4C expression in neural stem/progenitor cells and in adult neurogenesis induced by cerebral ischemia. J Mol Neurosci. 2009;39:27-39. 
22. Miyamoto E, Tomimoto H, Nakao Si S, Wakita H, Akiguchi I, Miyamoto K, et al. Caudoputamen is damaged by hypocapnia during mechanical ventilation in a rat model of chronic cerebral hypoperfusion. Stroke. 2001;32:2920-5.

23. Yang L, Tucker D, Dong Y, Wu C, Lu Y, Li Y, et al. Photobiomodulation therapy promotes neurogenesis by improving post-stroke local microenvironment and stimulating neuroprogenitor cells. Exp Neurol. 2018;299(Pt A):86-96.

24. Ai S, Fan X, Fan L, Sun Q, Liu Y, Tao X, et al. Extraction and chemical characterization of Angelica sinensis polysaccharides and its antioxidant activity. Carbohydr Polym. 2013;94:731-6.

25. Zhang $L$, Wang $H$, Wang $T$, Jiang $N$, Yu $P$, Chong $Y$, et al. Ferulic acid ameliorates nerve injury induced by cerebral ischemia in rats. Exp Ther Med. 2015;9:972-6.

26. Yi L, Liang Y, Wu H, Yuan D. The analysis of Radix Angelicae Sinensis (Danggui). J Chromatogr A. 2009;1216:1991-2001.

27. Ren Z, Zhang R, Li Y, Yang Z, Yang H. Ferulic acid exerts neuroprotective effects against cerebral ischemia/reperfusion-induced injury via antioxidant and anti-apoptotic mechanisms in vitro and in vivo. Int J Mol Med. 2017;40:1444-56.

28. Xin J, Zhang J, Yang Y, Deng M, Xie X. Radix Angelica Sinensis that contains the component Zligustilide promotes adult neurogenesis to mediate recovery from cognitive impairment. Curr Neurovasc Res. 2013;10:304-15.

29. Cheng CY, Kao ST, Lee YC. Angelica sinensis extract protects against ischemia-reperfusion injury in the hippocampus by activating p38 MAPK-mediated p90RSK/p-Bad and p90RSK/CREB/BDNF signaling after transient global cerebral ischemia in rats. J Ethnopharmacol. 2020; 2020:112612.

30. Pulsinelli WA, Brierley JB. A new model of bilateral hemispheric ischemia in the unanesthetized rat. Stroke. 1979;10:267-72.

31. Kuo CT, Lin YW, Tang NY, Cheng CY, Hsieh CL. Electric stimulation of the ears ameliorated learning and memory impairment in rats with cerebral ischemia-reperfusion injury. Sci Rep. 2016;6:20381.

32. Cheng CY, Ho TY, Hsiang CY, Tang NY, Hsieh CL, Kao ST, et al. Angelica sinensis Exerts Angiogenic and Anti-apoptotic Effects Against Cerebral Ischemia-Reperfusion Injury by Activating p38MAPK/HIF1[Formula: see text]/VEGF-A Signaling in Rats. Am J Chin Med. 2017;45:1683-708.

33. Cheng CY, Kao ST, Lee YC. Ferulic Acid Exerts Anti-apoptotic Effects against Ischemic Injury by Activating HSP70/Bcl-2- and HSP70/Autophagy-Mediated Signaling after Permanent Focal Cerebral Ischemia in Rats. Am J Chin Med. 2019;47:39-61.

34. Deb I, Manhas N, Poddar R, Rajagopal S, Allan AM, Lombroso PJ, et al. Neuroprotective role of a brain-enriched tyrosine phosphatase, STEP, in focal cerebral ischemia. J Neurosci. 2013;33:1781426.

35. Han H, Wu LM, Han MX, Yang WM, Wang YX, Fang ZH. Diabetes impairs spatial learning and memory and hippocampal neurogenesis via BDNF in rats with transient global ischemia. Brain Res Bull. 2016;124:269-77. 
36. Kempermann G, Song H, Gage FH. Neurogenesis in the Adult Hippocampus. Cold Spring Harb Perspect Biol. 2015;7:a018812.

37. Zhuang P, Zhang Y, Cui G, Bian Y, Zhang M, Zhang J, et al. Direct stimulation of adult neural stem/progenitor cells in vitro and neurogenesis in vivo by salvianolic acid B. PloS one. 2012;7:e35636.

38. Jiang W, Gu W, Brannstrom T, Rosqvist R, Wester P. Cortical neurogenesis in adult rats after transient middle cerebral artery occlusion. Stroke. 2001;32:1201-7.

39. Liu J, Solway K, Messing RO, Sharp FR. Increased neurogenesis in the dentate gyrus after transient global ischemia in gerbils. J Neurosci. 1998;18:7768-78.

40. Engelhard K, Winkelheide U, Werner C, Kluge J, Eberspacher E, Hollweck R, et al. Sevoflurane affects neurogenesis after forebrain ischemia in rats. Anesth Analg. 2007;104:898-903.

41. Tian L, Nie H, Zhang Y, Chen Y, Peng Z, Cai M, et al. Recombinant human thioredoxin-1 promotes neurogenesis and facilitates cognitive recovery following cerebral ischemia in mice. Neuropharmacology. 2014;77:453-64.

42. Song MD, Kim DH, Kim JM, Lee HE, Park SJ, Ryu JH, et al. Danggui-Jakyak-San ameliorates memory impairment and increase neurogenesis induced by transient forebrain ischemia in mice. BMC Complement Altern Med. 2013;13:324.

43. Schartz ND, Herr SA, Madsen L, Butts SJ, Torres C, Mendez LB, et al. Spatiotemporal profile of Map2 and microglial changes in the hippocampal CA1 region following pilocarpine-induced status epilepticus. Sci Rep. 2016;6:24988.

44. Jin K, Minami M, Lan JQ, Mao XO, Batteur S, Simon RP, et al. Neurogenesis in dentate subgranular zone and rostral subventricular zone after focal cerebral ischemia in the rat. Proc Natl Acad Sci U S A. 2001;98:4710-15.

45. Wang $\mathrm{C}$, Wang $\mathrm{H}$. Protective roles of heat stress on the neurons in hippocampal $\mathrm{CA} 1$ region of mice. Front Med China. 2007;1:418-22.

46. Zhao JJ, Hu JX, Lu DX, Ji CX, Qi Y, Liu XY, et al. Soluble cpg15 from Astrocytes Ameliorates Neurite Outgrowth Recovery of Hippocampal Neurons after Mouse Cerebral Ischemia. J Neurosci. 2017;37:1628-47.

47. Cheng CY, Lin JG, Tang NY, Kao ST, Hsieh CL. Electroacupuncture at different frequencies $(5 \mathrm{~Hz}$ and $25 \mathrm{~Hz}$ ) ameliorates cerebral ischemia-reperfusion injury in rats: possible involvement of p38 MAPKmediated anti-apoptotic signaling pathways. BMC Complement Altern Med. 2015;15:241.

48. Cheng CY, Tang NY, Kao ST, Hsieh CL. Ferulic Acid Administered at Various Time Points Protects against Cerebral Infarction by Activating p38 MAPK/p90RSK/CREB/Bcl-2 Anti-Apoptotic Signaling in the Subacute Phase of Cerebral Ischemia-Reperfusion Injury in Rats. PloS one. 2016;11:e0155748.

49. Ortega-Martinez S. A new perspective on the role of the CREB family of transcription factors in memory consolidation via adult hippocampal neurogenesis. Front Mol Neurosci. 2015;8:46.

50. Sasaki T, Kitagawa K, Omura-Matsuoka E, Todo K, Terasaki Y, Sugiura S, et al. The phosphodiesterase inhibitor rolipram promotes survival of newborn hippocampal neurons after 
ischemia. Stroke. 2007;38:1597-605.

51. Okuyama S, Shimada N, Kaji M, Morita M, Miyoshi K, Minami S, et al. Heptamethoxyflavone, a citrus flavonoid, enhances brain-derived neurotrophic factor production and neurogenesis in the hippocampus following cerebral global ischemia in mice. Neurosci Lett. 2012;528:190-5.

52. Lee JS, Hong SS, Kim HG, Lee HW, Kim WY, Lee SK, et al. Gongjin-Dan Enhances Hippocampal Memory in a Mouse Model of Scopolamine-Induced Amnesia. PloS one. 2016;11:e0159823.

53. Chen Y, Ai Y, Slevin JR, Maley BE, Gash DM. Progenitor proliferation in the adult hippocampus and substantia nigra induced by glial cell line-derived neurotrophic factor. Exp Neurol. 2005;196:87-95.

54. Geiseler SJ, Morland C. The Janus Face of VEGF in Stroke. Int J Mol Sci. 2018;19:1362.

55. Cao L, Jiao X, Zuzga DS, Liu Y, Fong DM, Young D, et al. VEGF links hippocampal activity with neurogenesis, learning and memory. Nat Genet. 2004;36:827-35.

\section{Tables}

Table 1. Primary antibodies used in this study

\begin{tabular}{|llllll|}
\hline Host & Primary antibody & IHC & IF & WB & Source/catalog No. \\
(Dilution) & $\begin{array}{l}\text { (Dilution) } \\
\text { (Dilution) }\end{array}$ & \\
\hline Rabbit & BrdU & & $1: 100$ & & abcam/ab152095 \\
\hline Mouse & NeuN & $1: 100$ & & Sigma-Aldrich/MAB377 \\
\hline Mouse & BrdU & $1: 100$ & & CST/\#5292 \\
\hline Rabbit & Ki67 & $1: 100$ & & abcam/ab15580 \\
\hline Rabbit & MAP-2 & $1: 100$ & & abcam/ab32454 \\
\hline Rabbit & p-p38 MAPK & & $1: 1000$ & CST/\#9211 \\
\hline Rabbit & P38 MAPK & & $1: 1000$ & CST/\#9212 \\
\hline Mouse & p-CREB & & $1: 500$ & Millipore/05-807 \\
\hline Rabbit & CREB & & $1: 1000$ & CST/\#9197 \\
\hline Rabbit & BDNF & & $1: 1000$ & abcam/ab108319 \\
\hline Rabbit & NGF & & $1: 1000$ & abcam/ab6199 \\
\hline Rabbit & GDNF & & $1: 500$ & abcam/ab18956 \\
\hline Rabbit & VEGF-A & & $1: 1000$ & Proteintech/\#19003-1-AP \\
\hline Mouse & Actin (loading control) & & $1: 5000$ & NB/NB600-501 \\
\hline
\end{tabular}


IHC, immunohistochemistry; IF, immunofluorescence; WB, Western blotting; CST, Cell Signaling Technology; NB, Novus Biologicals.

\section{Figures}

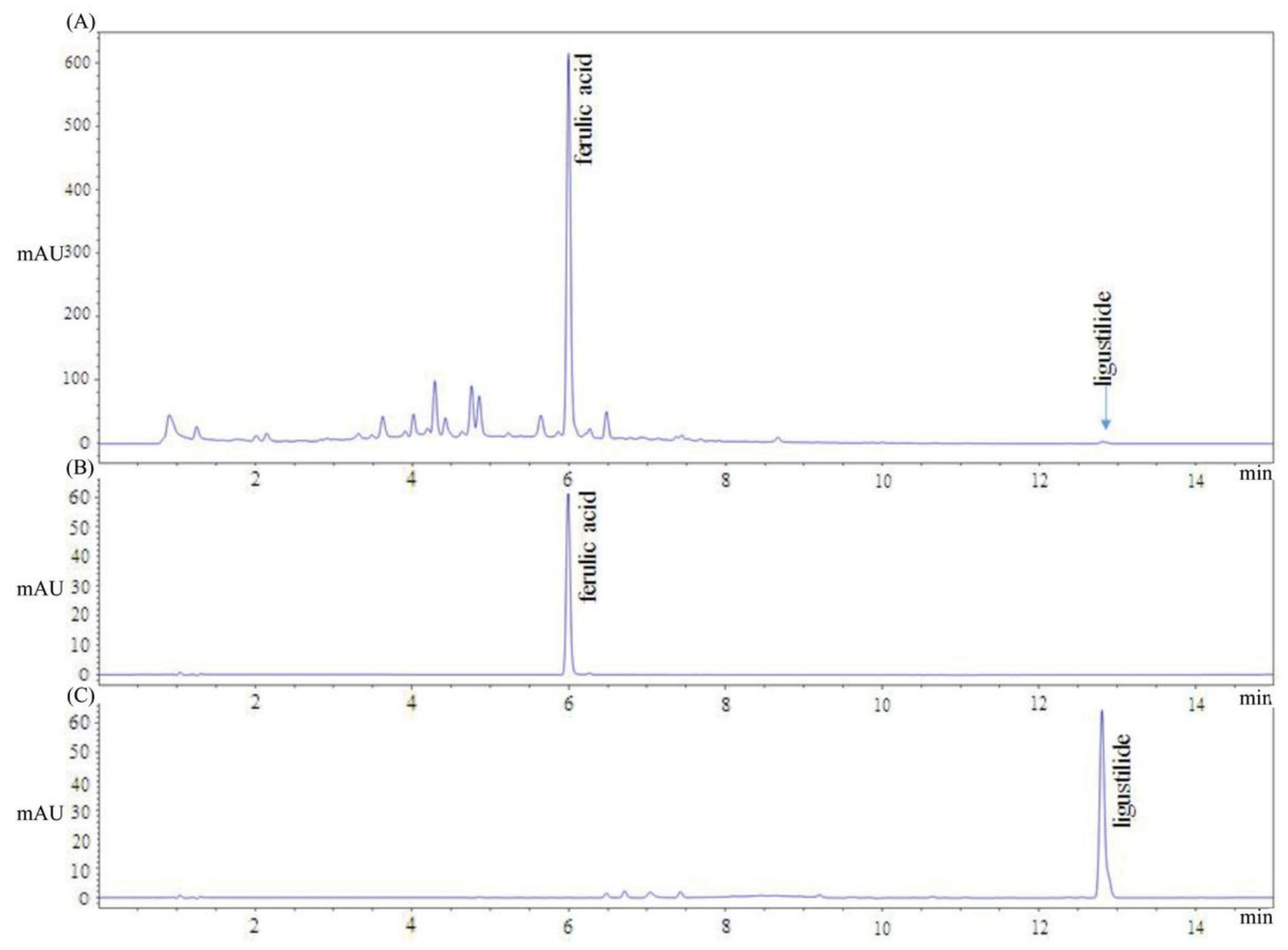

\section{Figure 1}

UPLC chromatograms concerning ferulic acid and ligustilide in the ASD extract. (A) The chemical profile of the ASD extract was performed using a C-18 column and detected at $320 \mathrm{~nm}$. UPLC chromatograms reveal the peak areas of $(B)$ ferulic acid $(t R=5.87 \mathrm{~min})$ and $(C)$ ligustilide $(t R=12.81 \mathrm{~min})$ standards. $\mathrm{mAU}$, mini absorbance unit. 


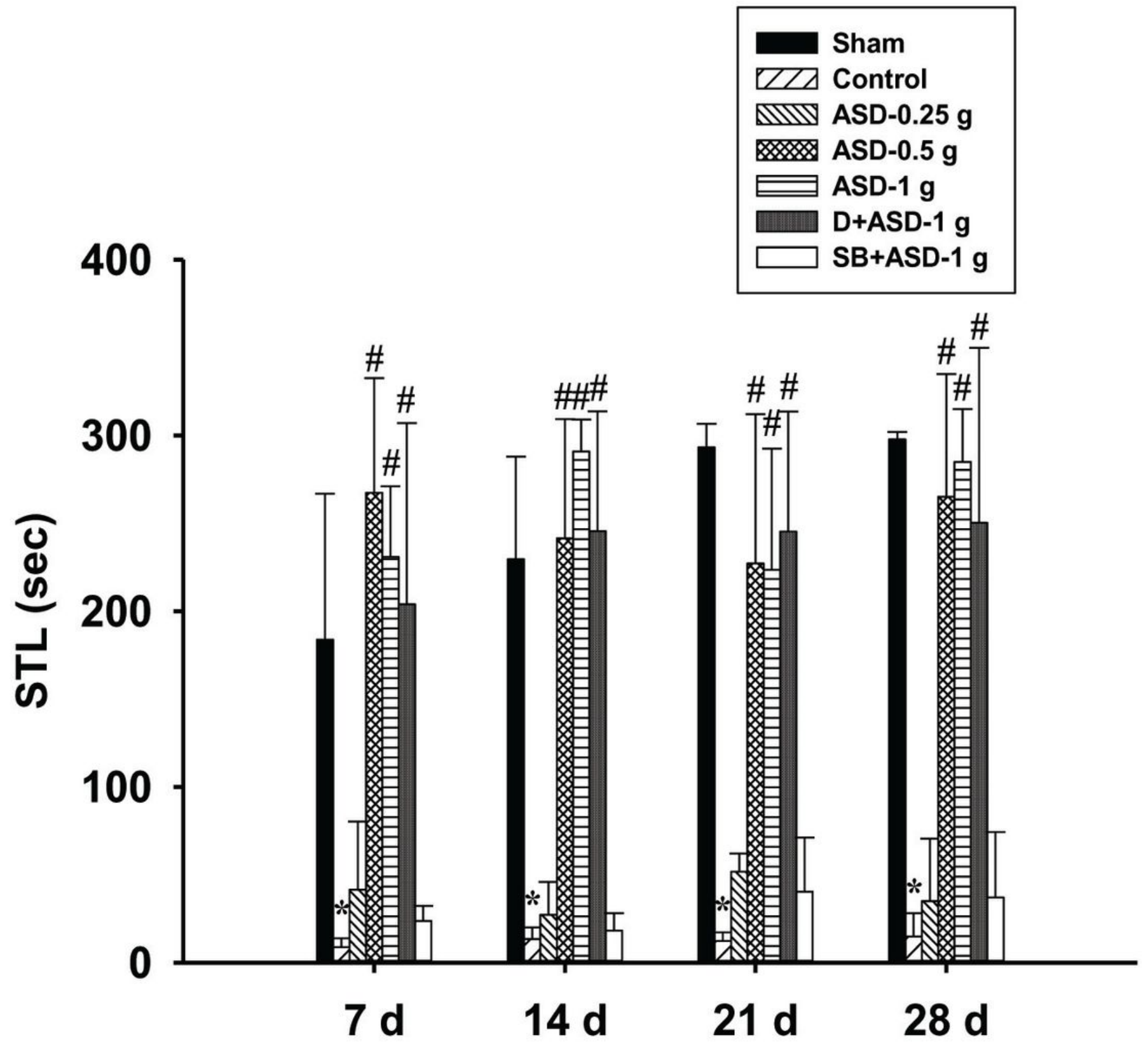

Figure 2

Effects of ASD extract treatments on memory impairment. The STLs of the Sham, Control, ASD- $0.25 \mathrm{~g}$, ASD-0.5 g, ASD-1 g, D+ASD-1 g, and SB+ASD-1 g groups $(n=4)$ were evaluated at 7, 14, 21, and $28 \mathrm{~d}$ after transient GCI. STL, step-through latency. Data are presented as mean \pm standard deviation. ${ }^{*} \mathrm{P}<0.05$ versus the Sham group; \#P 0.05 versus the Control group. 


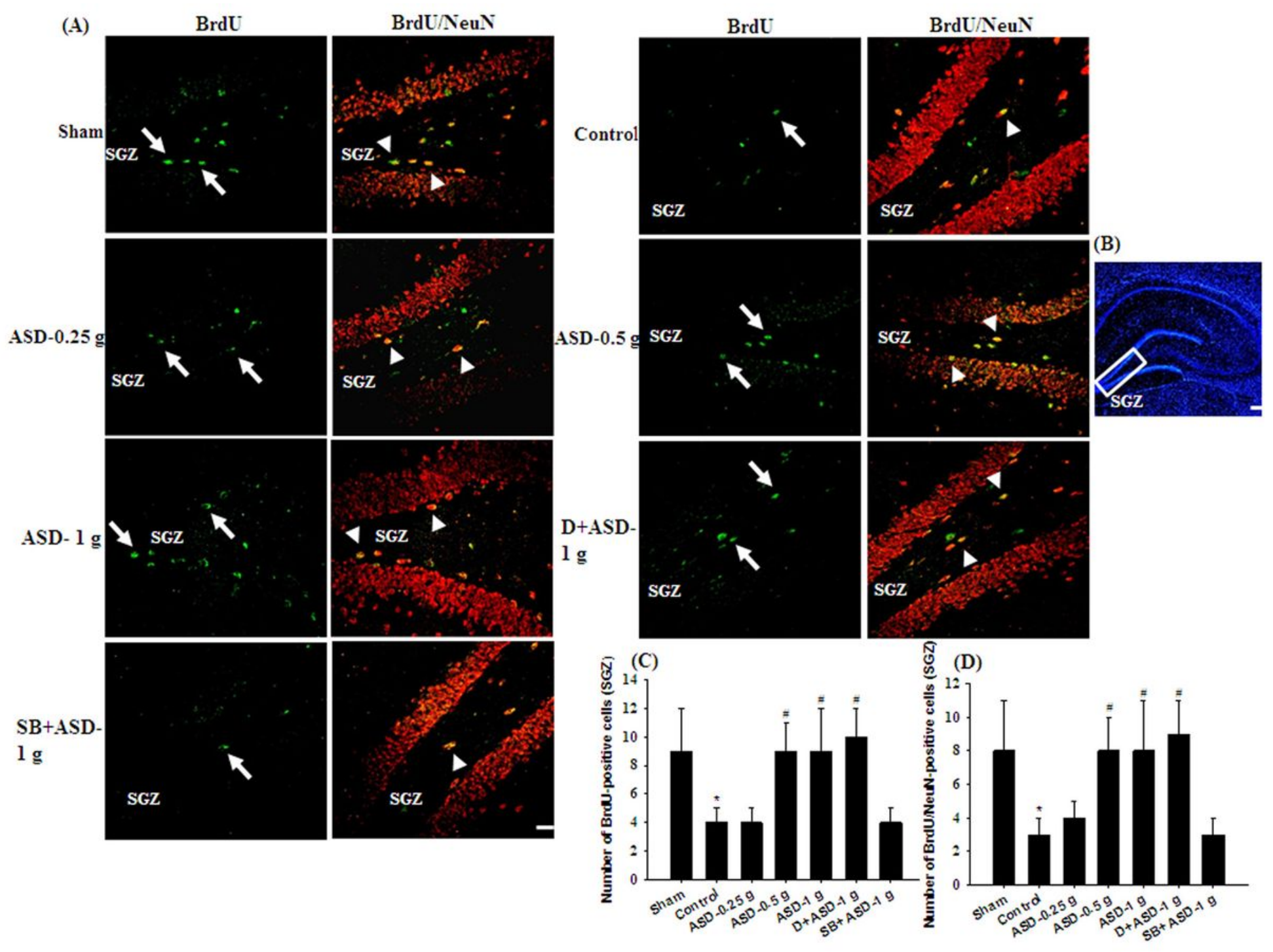

Figure 3

Effects of ASD extract treatments on hippocampal neurogenesis in the SGZ of the hippocampal DG. (A) The representative images reveal BrdU and BrdU/NeuN expression in the hippocampal SGZ in the Sham, Control, ASD-0.25 g, ASD-0.5 g, ASD-1 g, D+ASD-1 g, and SB+ASD-1 g groups $(n=4-5)$ at $28 \mathrm{~d}$ after transient $\mathrm{GCl}$. (B) The representative image provides a DAPI-stained section of the hippocampus. The tilted rectangle indicates the selected hippocampal SGZ, in which the immunopositive cells were calculated. The bar graphs illustrate the numbers of (C) BrdU-positive and (D) BrdU/NeuN-positive cells in the hippocampal SGZ in the experimental groups. ${ }^{*} \mathrm{P}<0.05$ versus the Sham group; $\# \mathrm{P}<0.05$ versus the Control group. Arrows and arrowheads indicate BrdU-positive and BrdU/NeuN-positive cells, respectively. Scale bars in (A) and (B) are equal to $50 \mu \mathrm{m}$ and $200 \mu \mathrm{m}$, respectively. 


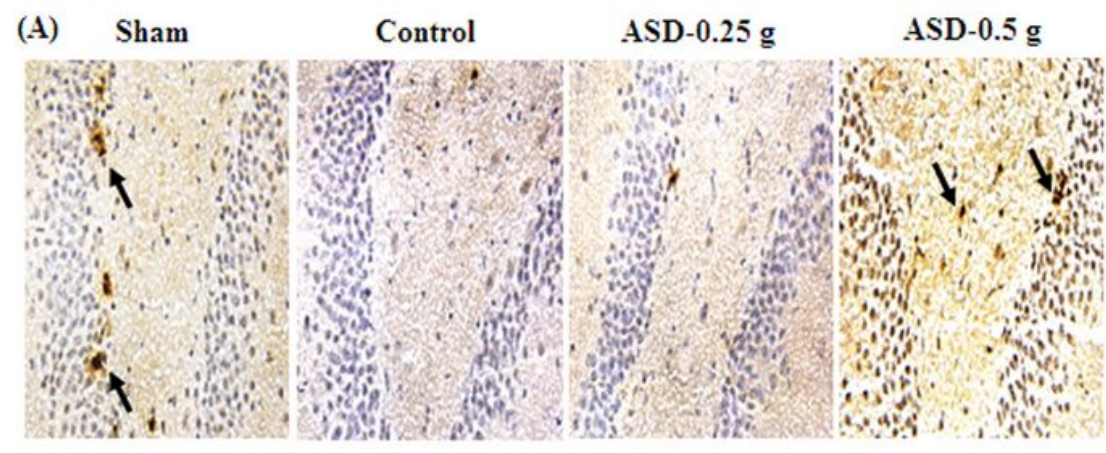

(B) Negative control

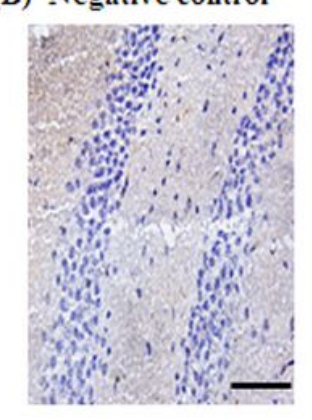

(E)

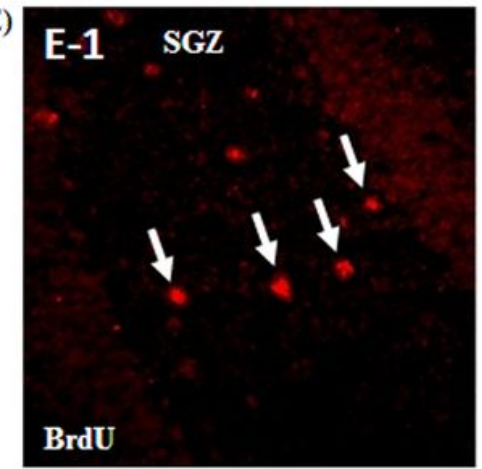

(C)

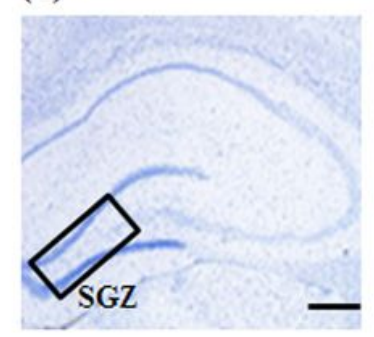

ASD-1 g

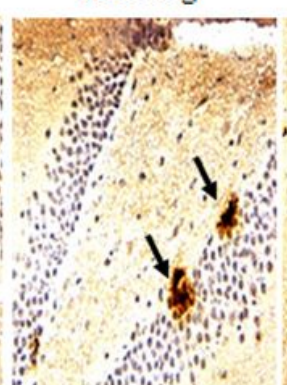

(D)
D+ASD-1 g

SB+ASD-1 g
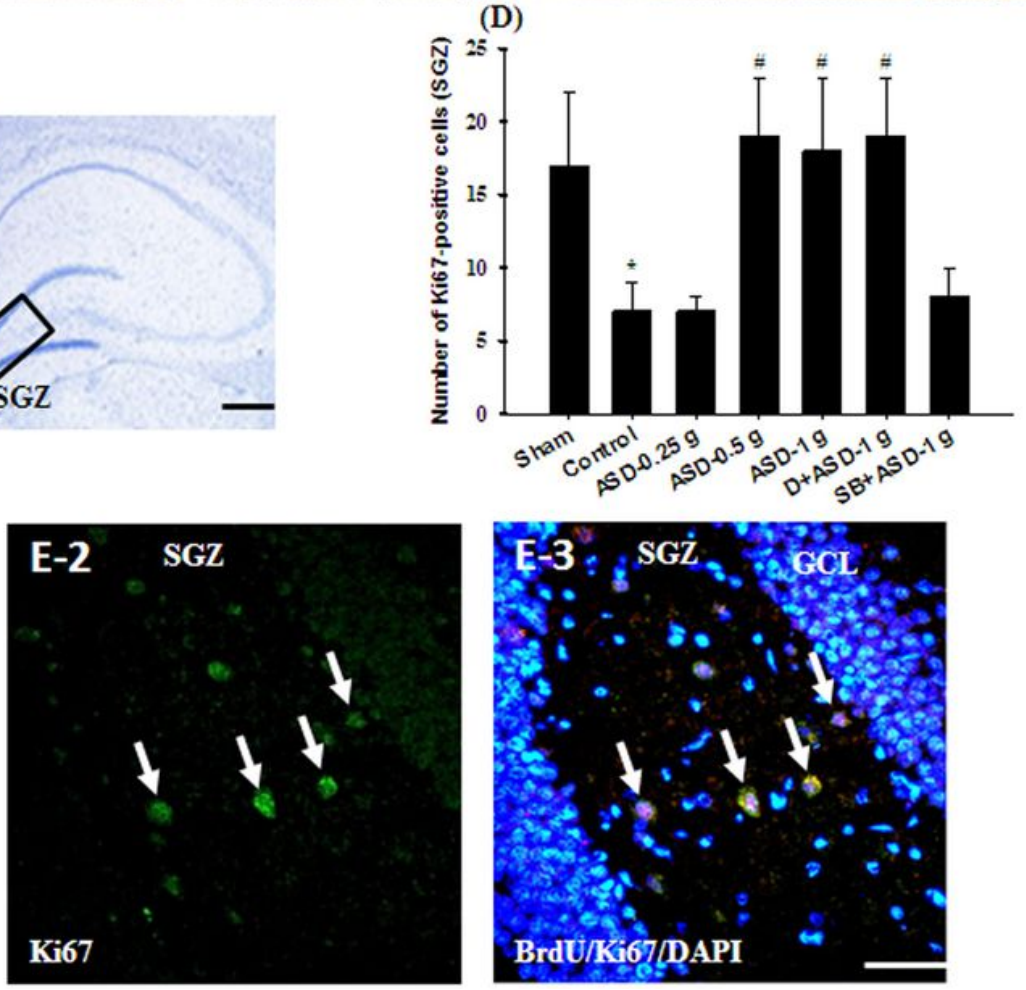

\section{Figure 4}

Effects of ASD extract treatments on Ki67 and BrdU/Ki67 expression in the hippocampus. (A) The representative images reveal ki67 expression in the hippocampal SGZ in the Sham, Control, ASD-0.25 g, ASD-0.5 g, ASD-1 g, D+ASD-1 g, and SB+ASD-1 g groups ( $n=4-5)$ at $28 \mathrm{~d}$ after transient GCl. (B) The representative image reveals the negative control for Ki67 antibody. (C) The tilted rectangle indicates the selected hippocampal SGZ, in which Ki67-positive cells were calculated. (D) The bar graph illustrates the number of Ki67-positive cells in the hippocampal SGZ in the experimental groups. ${ }^{*} P<0.05$ versus the Sham group; $\# \mathrm{P}<0.05$ versus the Control group. $(E)$ The representative images indicate BrdU-positive cells co-labeling with Ki67. Arrows in (A), (E-1), (E-2), and (E-3) indicate Ki67-positive, BrdU-positive (red), Ki67-positive (green), and BrdU/Ki67-positive (yellow orange) cells, respectively. Scale bars in (A), (B), (C), and (E-3) are equal to $10 \mu \mathrm{m}, 10 \mu \mathrm{m}, 500 \mu \mathrm{m}$, and $50 \mu \mathrm{m}$, respectively. 


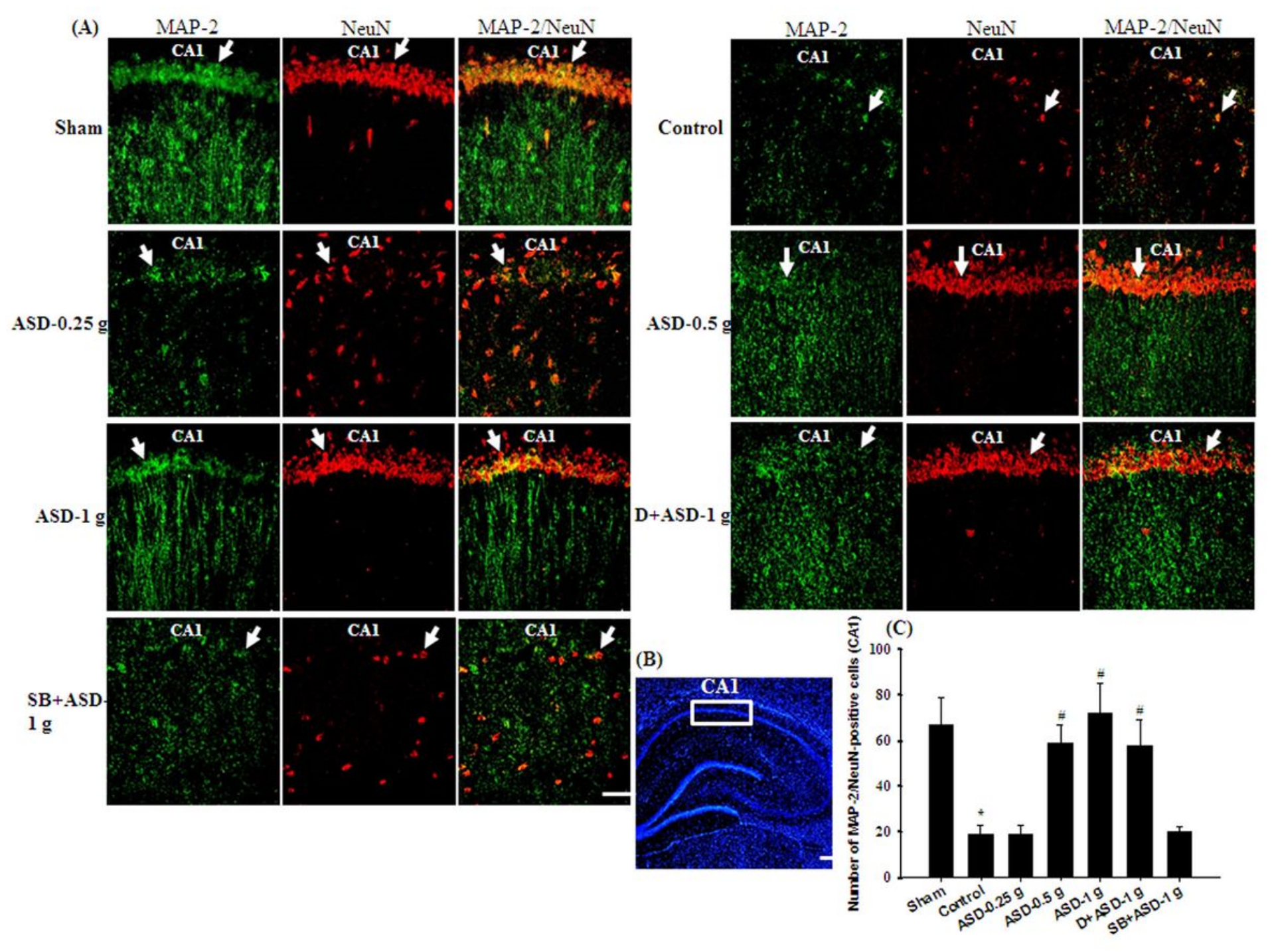

Figure 5

Effects of ASD extract treatments on MAP-2/NeuN expression in the hippocampus. (A) The representative images reveal MAP-2/NeuN expression in the CA1 region in the Sham, Control, ASD-0.25 g, ASD-0.5 g, ASD-1 g, D+ASD-1 g, and SB+ASD-1 g groups $(n=4-5)$ at $28 \mathrm{~d}$ after transient GCl. Intact MAP-2-positive dendrites were found in the Sham, ASD-0.5 g, ASD-1 g, and D+ASD-1 groups, whereas discontinuous patterns of MAP-2-positive dendrites were found in the Control, ASD-0.25 g, and SB+ASD-1 g groups. (B) The rectangle indicates the selected CA1 region, in which the MAP-2/NeuN-positive cells were calculated. (C) The bar graph illustrates the number of MAP-2/NeuN-positive cells in the CA1 region in the experimental groups. ${ }^{*} \mathrm{P}<0.05$ versus the Sham group; $\# \mathrm{P}<0.05$ versus the Control group. Arrows on the left (first and fourth columns), middle (second and fifth columns), and right (third and sixth columns) sides of the representative images indicate the MAP-2-positive (green), NeuN-positive (red), and MAP2/NeuN-positive (yellow orange) cells, respectively. Scale bars in (A) and (B) are equal to $50 \mu \mathrm{m}$ and 200 $\mu \mathrm{m}$, respectively. 
(A) Sham Control

A

p-p38 MAPK

p38 MAPK

p-CREB

CREB

BDNF

Actin
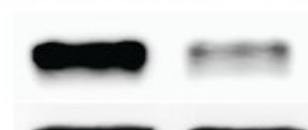
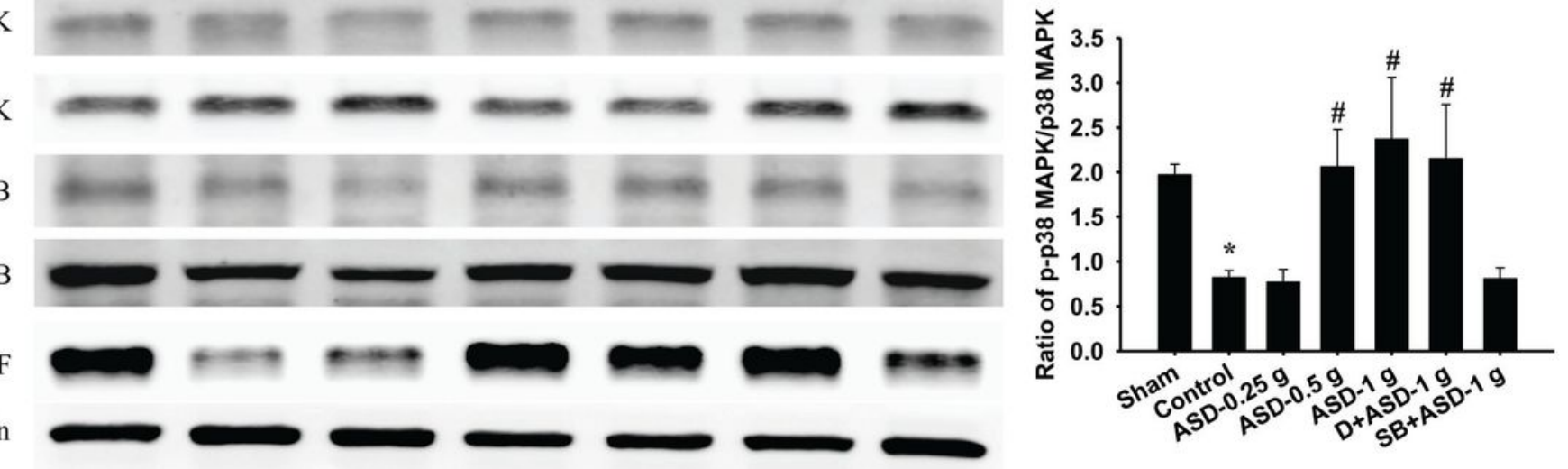

(C)

(D)
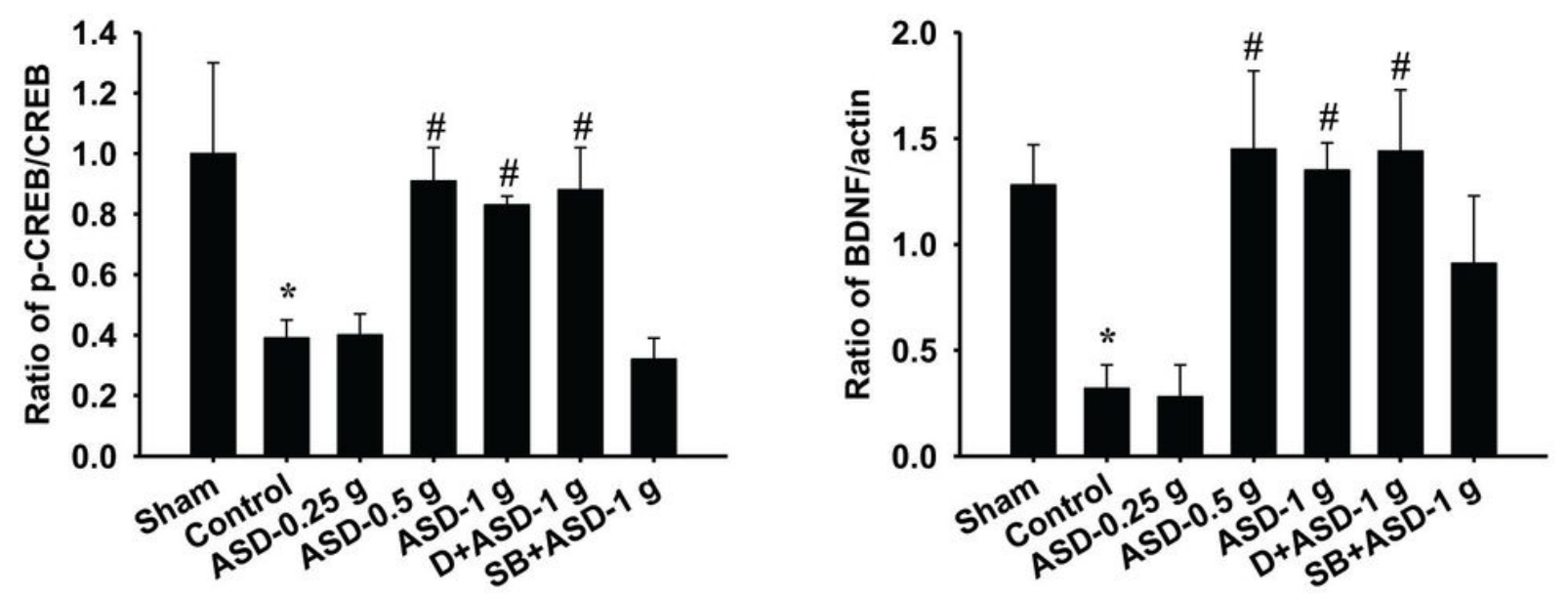

Figure 6

Effects of ASD extract treatments on p-p38 MAPK, p38 MAPK, p-CREB, CREB, and BDNF expression in the hippocampus. (A) The representative Western blot images reveal p-p38 MAPK, p38 MAPK, p-CREB, CREB, and BDNF expression in the hippocampus in the Sham, Control, ASD-0.25 g, ASD-0.5 g, ASD-1 g, D+ASD-1 $\mathrm{g}$, and SB+ASD-1 g groups $(n=4)$ at $28 \mathrm{~d}$ after transient $\mathrm{GCl}$. The ratios of $(\mathrm{B}) \mathrm{p}-\mathrm{p} 38 \mathrm{MAPK} / \mathrm{p} 38 \mathrm{MAPK}$, (C) $p$-CREB/CREB, and (D) BDNF/actin expression were evaluated in the hippocampus in the experimental groups. ${ }^{*} \mathrm{P}<0.05$ versus the Sham group; $\# \mathrm{P}<0.05$ versus the Control group. 
(A) Sham Control ASD-0.25 g ASD-0.5 g ASD-1 g D+ASD-1 g SB+ASD-1 g

(B)
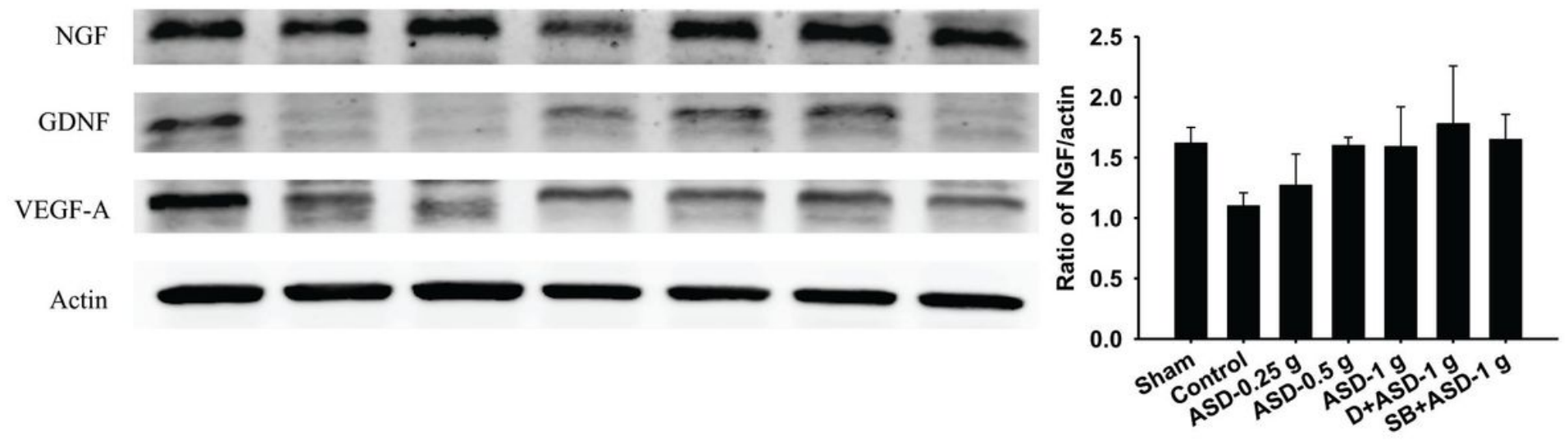

(C)

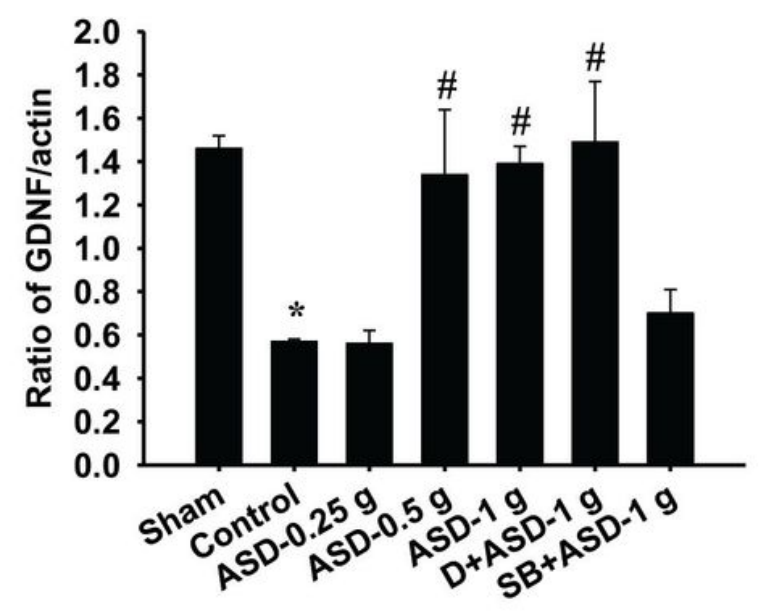

(D)

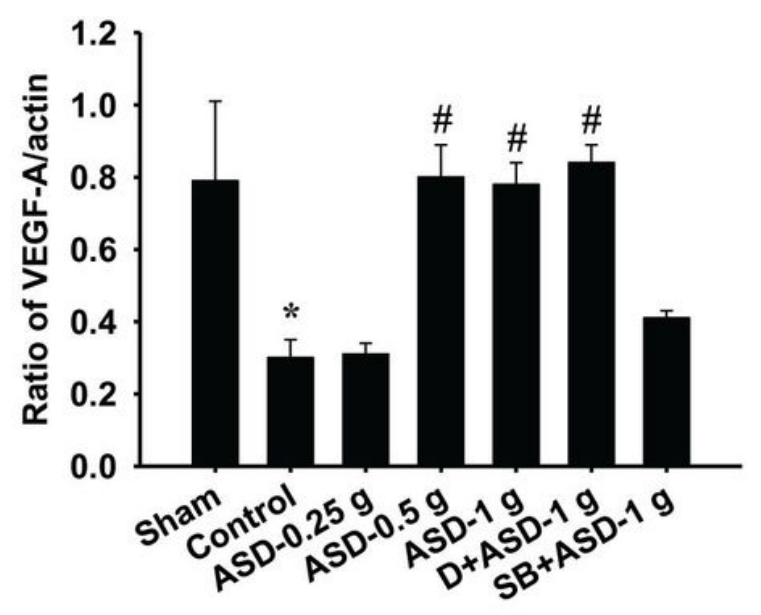

Figure 7

Effects of ASD extract treatments on NGF, GDNF, and VEGF-A expression in the hippocampus. (A) The representative Western blot images reveal NGF, GDNF, and VEGF-A expression in the hippocampus in the Sham, Control, ASD-0.25 g, ASD-0.5 g, ASD-1 g, D+ASD-1 g, and SB+ASD-1 g groups $(n=4)$ at $28 \mathrm{~d}$ after transient GCI. The ratios of (B) NGF/actin, (C) GDNF/actin, and (D) VEGF-A/actin expression were evaluated in the hippocampus in the experimental groups. ${ }^{*} P<0.05$ versus the Sham group; $\# P<0.05$ versus the Control group. 